Zum Artikel „Es gibt ein Recht auf ein ungesundes Leben“, ergopraxis 5/15

\section{Uns steht es nicht zu, Moralapostel zu sein}

Das Interview mit Prof. Gugutzer zum Thema Grenzen der Selbstoptimierung spricht mir aus dem Herzen. Noch nie konnte ich verstehen, warum ich joggen soll. Ich fühle mich während der sportlichen Aktion und auch danach nicht gut. In Ruhe ein Buch zu lesen, ins Kino oder Theater zu gehen, ist doch viel besser!

Bei den Klienten ist es genau das Gleiche. Klar kann ich alles therapeutisch Sinnvolle anpreisen, aber es gibt so viele innere und äußere Umstände, warum es nicht umgesetzt wird. Und hier steht es mir nicht zu, mich als Moralapostel aufzuführen, zu strafen und zu prophezeien: „Wenn Sie das nicht so oder so machen, werden Sie nie gesund." Jeder muss lernen, mit seiner Erkrankung und seinen Handicaps zu leben und einen eigenen Weg zu finden. Manchmal eben auch mit Schokolade und Chips. So wie eine Klientin, die bei mir wegen ihrer Hemiplegie rechts in Behandlung ist. Sie erlitt im Mai 2013 einen Apoplex. Sie nimmt zwar zu und wird nun noch ungelenker werden, freut sich aber an kleinen Fortschritten und hat vor allem ihren Humor wiedergefunden. Sie lacht wieder! Und das soll ja auch gesund sein.

Mit freundlichen Grüßen

Hiltrud Gröss, Ergotherapeutin aus Calw

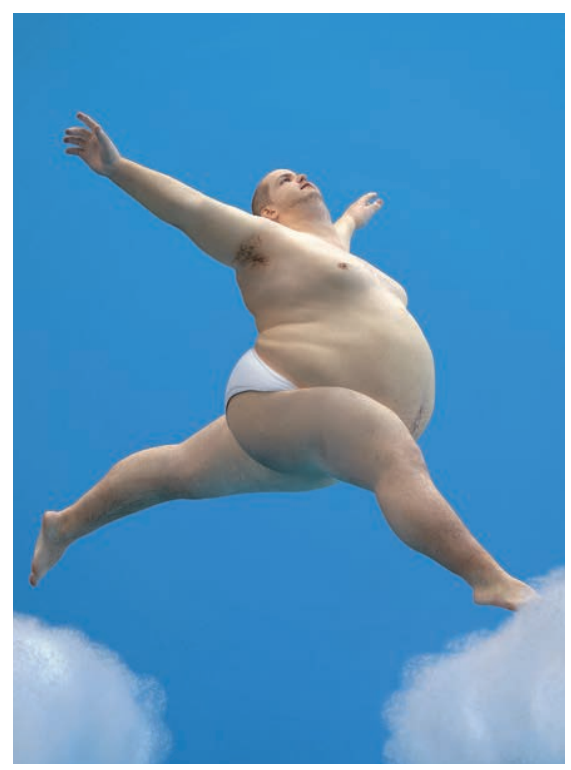

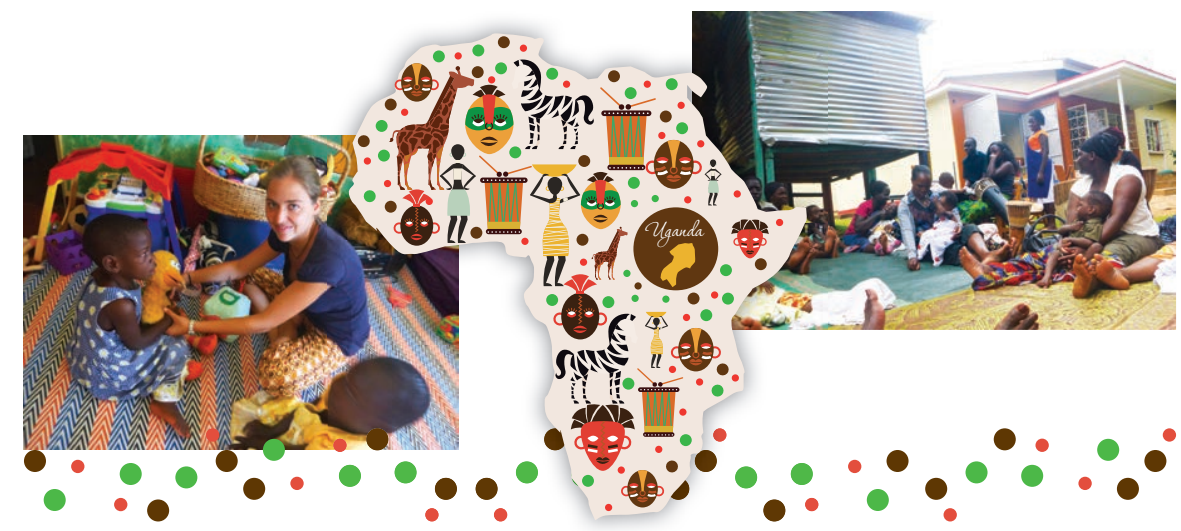

Zum Artikel „Eine Ergotherapeutin in Uganda“, ergopraxis 11-12/14

\section{Faszinierend}

Der Bericht von Sonja Pfirsching über ihre Erfahrungen als Ergotherapeutin in Uganda hat mich sehr fasziniert. Ich bin zurzeit in der Ausbildung an der paritätischen Berufsfach-

Erratum zum Artikel „Erste promovierte Ergotherapeutin in der Schweiz“, ergopraxis 4/15

\section{Erste Promovierte im Fach Ergotherapie}

Es mag auf den ersten Blick spitzfindig erscheinen, doch der Unterschied ist bedeutend: Brigitte Gantschnig ist nicht die „Erste promo- schule Hausach als Ergotherapeutin und werde im Juli 2015 meinen Abschluss machen. Daraufhin habe ich vor, ins Ausland zu gehen, bevor ich richtig anfangen werde, als Ergo zu arbeiten. Danke für diese Inspiration.

Liebe Grüße Jessica Moosmann aus Lauterbach im Schwarzwald

vierte Ergotherapeutin in der Schweiz", sondern sie ist die erste, die im Fach Ergotherapie promoviert hat. In der Schweiz gibt es bereits promovierte Ergotherapeuten, die ihren Doktortitel allerdings in anderen Fachbereichen erworben haben. Die Darstellung in ergopraxis 4/15 auf Seite 12 könnte also falsch verstanden werden. Das möchten wir hiermit richtigstellen.

Beste Grüße das Team der ergopraxis $\rightarrow$ SCHREIBEN SIE UNS

\section{Ihre Meinung zählt}

Mehr Wissen, mehr Austausch, mehr Erfolg - dafür engagieren sich unsere Autoren und das Team der ergopraxis. Auch Sie sind eingeladen, bei uns mitzuwirken. Ihre Meinungen, Kommentare und Anregungen sind bei ergopraxis jederzeit willkommen!

Georg Thieme Verlag KG

Redaktion ergopraxis

Rüdigerstraße 14, 70469 Stuttgart

Fax: 0711/8931-874

E-Mail: ergopraxis@thieme.de

\title{
www.thieme.de/ ergopraxis
}




\section{Das Artikelarchiv von ergopraxis}

Wer lieber elektronisch liest oder bereits erschienene Artikel sucht, ist im Artikelarchiv der ergopraxis richtig. Dort stehen für Sie alle Beiträge, Literaturverzeichnisse und Zusatzmaterialien zum Herunterladen bereit. Gehen Sie dazu einfach ins Internet zu www.thieme-connect.de/products/ergopraxis.

Auf der Startseite unseres Artikelarchivs präsentieren wir Ihnen immer die aktuelle Ausgabe.

Über die Menüpunkte links unter dem Titelbild kommen Sie über die Jahreszahl und die Wahl der gewünschten Ausgabe zu den einzelnen Ausgaben und Artikeln.

Wer auf der Suche nach einem bestimmten Beitrag oder Thema ist, wird über die Volltextsuche rechts oben fündig.

Reinschauen lohnt sich. In unserem Artikelarchiv wartet ein Fundus aus sieben Jahren ergopraxis auf Sie.

Um auf das Archiv zugreifen zu können, müssen Sie sich einmalig registrieren lassen. Dazu klicken Sie auf der oben genannten Startseite auf den Button „Jetzt registrieren“ und wählen Sie die Option „Registrierung für persönliche Abonnenten“ aus. Am Ende des Registrierungsprozesses geben Sie unter ergopraxis Ihre Abonummer

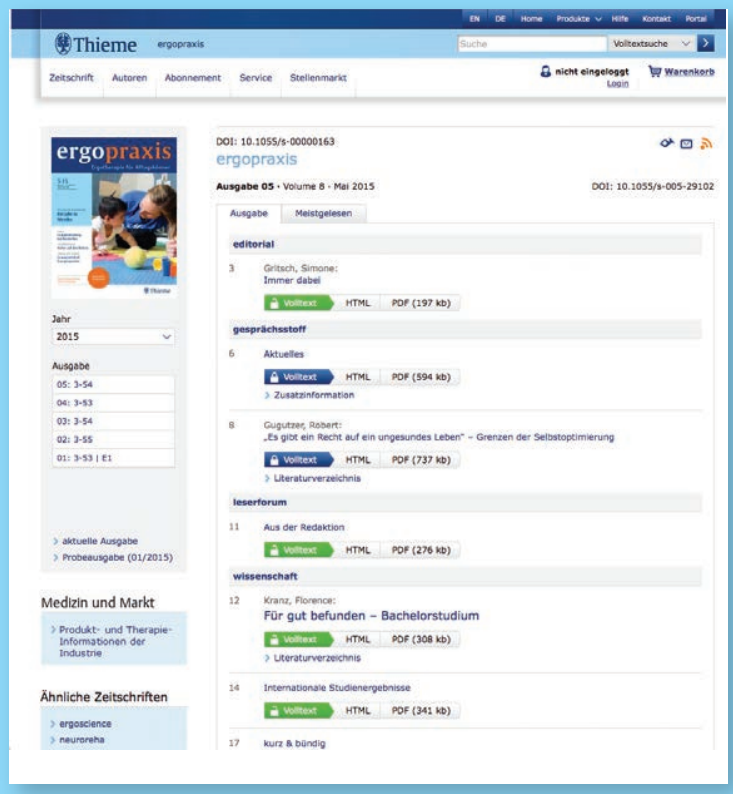
ein. Das sind die 6 oder 7 Ziffern auf Ihrem Adressetikett (o unten).

Nach erfolgreicher Registrierung haben Sie zunächst Zugriff auf den aktuellen Jahrgang der ergopraxis. Das Abonnement wird überprüft und der Zugang dann auch für die zurückliegenden Jahrgänge freigeschaltet.

Beim nächsten Aufruf des Artikelarchiv der ergopraxis brauchen Sie für das Login nun nur Ihren Benutzernamen und Ihr Passwort in die entsprechenden Felder einzutragen. Dann haben Sie als Abonnent Zugriff auf alle Artikel der ergopraxis.

Wer sich über Neuerscheinungen der ergopraxis online benachrichtigen lassen möchte, kann den Alert-Service nutzen oder den RSS-Feed-Button auf der Startseite aktivieren.

\section{ZU GEWINNEN}

$3 \times$ Heilmittel-Telegramm 04/2014 und Heilmittelrichtlinie

3 x Ratgeber „Die 50 besten Rückenschmerzkiller“ 3 x zwei Noppenbälle

$1 \mathrm{x}$ Jahresabo neuroreha

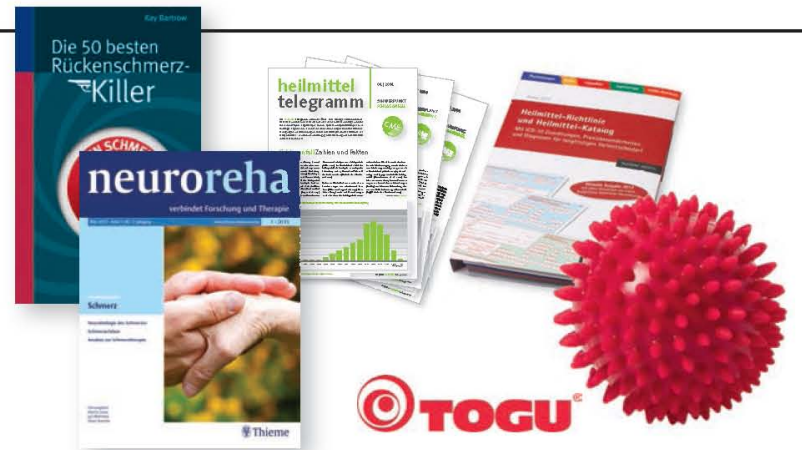

Wir gratulieren allen Gewinnern aus der Ausgabe 4/2015:

Petra Sörensen, Hamburg, Christina Gotzian, Dankmarshausen, Lina Taplick, Berlin, Sarah Schlemaier, Ellmau (Österreich), Corinna Ahrens-Zehetner, Buchkirchen (Österreich), Annika Grauschopf, Nordholz, Jennifer Bernecker, Riemerling. Katja Wegehaupt, Berlin
Dank Sponsoren gibt es fur unsere Abonnenten in jeder ergopraxis etwas zu gewinnen. Kästen innerhalb der Artikel weisen auf die Gewinnspiele hin. Klicken Sie einfach bis

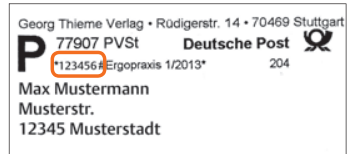
zum Ablauf der Frist unter www.thieme.de/

ergopraxis > "Gewinnspiel“ auf das gewünschte Stichwort und nennen Sie uns Ihre Abonummer. Ihre Abonummer besteht aus den 6 oder 7 Ziffern auf Ihrem Adressetikett.

Wir drücken Ihnen die Daumen!
Seite 11

Seite 21

Seite 31 